Ricardo de Carvalho CaValu'

ValérIa CRISTINA SANDRIM ${ }^{2}$

José Eduardo Tanus dos Santos ${ }^{3}$

Geraldo Duarte ${ }^{4}$

\section{Predição de pré-eclâmpsia}

\author{
Preeclampsia predition
}

\section{Introdução}

Pré-eclâmpsia é uma condição específica da gestação que envolve a falência de diversos órgãos e está associada à hipertensão e proteinúria. $\mathrm{O}$ aumento da pressão sanguínea provoca efeitos deletérios sobre diversos sistemas, principalmente o vascular, o hepático, o renal e o cerebral. As complicações observadas nesses sistemas podem explicar a alta incidência de mortalidade e morbidade fetal e materna, o que faz da pré-eclâmpsia uma das principais causas de morte materna no Brasil (37\% das causas de morte obstétricas diretas) ${ }^{1}$ e em vários outros países ${ }^{2}$. A frequência de pré-eclâmpsia varia de 2 a $10 \%$ das gestações em todo o mundo ${ }^{3,4}$. É interessante ressaltar que existem alguns fatores de risco que aumentam a probabilidade de uma gestante apresentar pré-eclâmpsia como, por exemplo: hipertensão e diabetes mellitus pré-existentes, obesidade e a etnia. Apesar da importância óbvia do ponto de vista de saúde pública, a etiologia subjacente a essa condição permanece desconhecida.

Apesar da fisiopatologia da pré-eclâmpsia ainda ser desconhecida, é amplamente aceito, atualmente, o fato de que a isquemia da placenta é um fator primordial. Durante o início do segundo trimestre da gestação (entre a $18^{\mathrm{a}}$ e $20^{\mathrm{a}}$ semana) instala-se um processo referido como "pseudovasculogenese", caracterizado pela migração dos citotrofoblastos em direção às arteríolas uterinas espiraladas onde sofrem diferenciação em células com fenótipo endotelial. Nesse processo, ocorre remodelamento gradual da camada endotelial desses vasos e destruição do tecido elástico-muscular das artérias e arteríolas, tornando-as mais dilatadas ${ }^{5-8}$. Essa migração/diferenciação dos citotrofoblastos deve-se a alterações nos perfis de expressão de certas citocinas, moléculas de adesão, constituintes da matrix extracelular, metaloproteinases e o antígeno de histocompatibilidade (HLA-G $)^{9-11}$. O remodelamento das artérias uterinas espiraladas resulta na formação de um sistema local de baixa resistência arteriolar que é essencial ao aumento do suprimento sanguíneo para o desenvolvimento e crescimento do feto. Na pré-eclâmpsia, a invasão das artérias espiraladas do útero é limitada, sendo que apenas entre 30 e $50 \%$ das artérias sofrem a invasão do trofoblasto. A média do diâmetro das artérias espiraladas de gestantes com pré-eclâmpsia é metade daquela observada na gravidez normal $^{12}$. Essa falência do remodelamento vascular impede uma resposta adequada ao aumento da demanda do fluxo sanguíneo que ocorre durante a gestação, diminuindo a perfusão útero-placentária e provocando isquemia da placenta. Mas qual seria a origem da
' Professor do Departamento de Ginecologia e Obstetrícia da Faculdade Medicina de Ribeirão Preto da Universidade de São Paulo - USP - Ribeirão Preto (SP), Brasil.

2 Pós-doutoranda do Departamento de Farmacologia da Faculdade Medicina de Ribeirão Preto da Universidade de São Paulo - USP - Ribeirão Preto (SP), Brasil.

${ }^{3}$ Professor do Departamento de Farmacologia da Faculdade Medicina de Ribeirão Preto da Universidade de São Paulo - USP Ribeirão Preto (SP), Brasil.

4 Professor Titular do Departamento de Ginecologia e Obstetrícia da Faculdade Medicina de Ribeirão Preto da Universidade de São Paulo - USP - Ribeirão Preto (SP), Brasil. 
pré-eclâmpsia? Por que em algumas mulheres a migração e diferenciação dos citotrofoblastos estão comprometidas? Essa pergunta continua sem resposta. Sugere-se que fatores maternos relacionados à predisposição genética, à adaptação imunológica à gravidez e doenças vasculares pré-existentes possam estar envolvidas nessa doença. É provável que haja diversas etiologias ou predisposições da doença com efeitos que resultam num grupo comum de sinais e sintomas que a caracteriza.

Muitos estudos, desde a década de 1990 até o presente momento, avaliaram a invasão trofoblástica por meio do estudo doplervelocimétrico das artérias uterinas, calculando-se o índice de pulsatilidade e a presença de incisura protodiastólica. Partia-se do pressuposto que a má-adaptação é causada pelos mesmos fatores responsáveis pela manutenção do alto índice de resistência das artérias uterinas e este, portanto, seria um fator preditor de desordens hipertensivas na gravidez ${ }^{13,14}$. Porém, na maior parte das avaliações, mesmo controlando-se fatores de confusão, não se encontrou sensibilidade mínima para tornar essa avaliação preditora do fenômeno da pré-eclâmpsia, não sendo conveniente o seu uso na prática clínica ${ }^{15,16}$. A partir desses estudos, passou-se a discutir que um provável fator endotelial se associa à má-adaptação, levando ao desenvolvimento da pré-eclâmpsia.

Nesse sentido e, tradicionalmente, a predição da pré-eclâmpsia tem sido baseada na detecção de fatores de risco da gestante, bem como a avaliação da pressão sanguínea, proteinúria e edema. Entretanto, várias gestantes que não apresentam tais fatores de risco também desenvolvem pré-eclâmpsia, o que torna necessária a obtenção de marcadores bioquímicos que podem predizer tal condição. Vários estudos tiveram como objetivo a identificação de marcadores. Entretanto, nenhum deles apresenta, atualmente, valor clínico. A importância da predição de quais mulheres desenvolverão pré-eclâmpsia é claramente necessária, pois direcionaria cuidados médicos especiais e medidas preventivas que poderiam prolongar a gestação e diminuir os riscos maternos e fetais.

Estudos familiares demonstram que de 25 a $31 \%$ de filhas de mulheres com pré-eclâmpsia desenvolvem a doença. Em filhas de mulheres sem o antecedente, esse valor varia de 5 a $10 \%{ }^{17}$. Nesse aspecto, existem fortes evidencias do componente genético na etiologia da pré-eclâmpsia, justificando os estudos baseados na predição genética e proteômica.

O genoma humano reflete o estudo do sequenciamento de genes do ser humano em todos os seus aspectos e permite a avaliação de alterações em pares de bases e a susceptibilidade a doenças adquiridas ${ }^{18}$. As principais alterações encontradas referem-se a deleções, inserções e duplicações de pares de bases, assim como podem também estar presentes polimorfismos de base única do $\mathrm{DNA}^{18}$. A presença dos polimorfismos genéticos podem, consequentemente, alterar a transcrição gênica, alterando também a estabilidade do RNAm com modificações nas concentrações proteicas e nas atividades enzimáticas, identificando, portanto, a susceptibilidade genética à pré-eclâmpsia.

No sequenciamento do genoma humano foram identificados muitos genes associados a um amplo espectro de doenças. Para a seleção de genes candidatos ao envolvimento com uma determinada doença, devem ser considerados os mecanismos envolvidos na fisiopatologia. Como na pré-eclâmpsia a disfunção endotelial está fortemente envolvida, muitos estudos têm sido conduzidos com o foco em genes envolvidos na manutenção da função do endotélio ${ }^{18}$.

O principal regulador do tônus vascular é o óxido nítrico (NO). No endotélio, esse gás é sintetizado pela sintetase endotelial do óxido nítrico (eNOS) por meio da conversão do aminoácido L-arginina e oxigênio em L-citrulina e $\mathrm{NO}^{19}$. Vários trabalhos vêm demonstrando que a modulação da via arginina/NO/GMPc tem um papel importante na gravidez ${ }^{20,21 .}$

De maneira geral, durante uma gestação normal é observado um aumento dos níveis de NO pela atividade da eNOS $^{22}$. A causa específica desse aumento é desconhecida, mas sugere-se que o aumento do shear stress ${ }^{23}$ e de certos hormônios, tais como o 17- $\beta$-estradiol ${ }^{24}$ e o $\mathrm{Hcg}^{25}$, durante a gravidez estimulem a atividade dessa enzima. Especificamente na placenta, a atividade da eNOS é importante no sentido de que o NO sintetizado mantém localmente a resistência vascular baixa, além de atenuar a ação dos vasoconstritores ${ }^{26}$.

Estima-se que $70 \%$ dos níveis sistêmicos sejam derivados da atividade da eNOS, além de que se sugere que esse composto seja um reservatório de NO na circulação, no qual sua conversão para NO se dá devido ao pH baixo ou a pressões de oxigênio baixas ${ }^{27}$. Dessa maneira, a quantificação dos nitritos plasmáticos seria mais indicada para análise da atividade da eNOS e, portanto, da produção endógena de NO.

Nesse contexto, foi avaliado o polimorfismo do gene do óxido nítrico sintetase (enos) em um grupo de mulheres com pré-eclâmpsia e em grupos de gestantes saudáveis. Observou-se menor frequência do alelo T Glu do gene da enos em pacientes com pré-eclâmpsia, o que sugere contribuição dos haplótipos da enos no desenvolvimento de desordens hipertensivas durante a gravidez ${ }^{28}$. A pré-eclâmpsia é uma doença multifatorial e, portanto, um número grande de genes pode estar envolvido. Quanto a essse aspecto, a expectativa em relação aos estudos genéticos baseia-se nas técnicas de microarray que permite análise global da expressão de milhares de genes, mas com custos elevados para uso corriqueiro. Como esses estudos precisam incluir um grande numero de sujeitos para que sejam encontradas as diferenças 
esperadas, o custo tem inviabilizado a sua execução ${ }^{29}$. Estudos genéticos, portanto, apresentam custo muito elevado e com provável dificuldade de inserção na prática clínica da predição das doenças hipertensivas a curto prazo.

A proteomica é o estudo de proteínas em grande escala que, em particular, estuda sua estrutura e função ${ }^{30,31}$. Essa tecnologia emergiu como uma importante ferramenta em muitos campos da pesquisa médica. Vem sendo usada, em particular, na identificação de biomarcadores que podem auxiliar no diagnóstico e avaliar o estágio de doenças cardiovasculares, renais e neurológicas ${ }^{31}$. A análise de substâncias presentes nos fluidos corpóreos asseguraria uma fonte de muitas informações e permitiriam monitorar o bem-estar do indivíduo. Nesse sentido, a urina, por apresentar proteínas e peptídeos fragmentados ${ }^{33}$ representativos dos que estão presentes na corrente sanguínea ${ }^{32,33}$, pode ser uma alternativa para pesquisas. Estudando o padrão de proteínas presentes na urina de gestantes ao longo da gravidez, será possível caracterizar marcadores proteicos específicos daquelas que irão desenvolver pré-eclâmpsia. A caracterização individual estabelecida com o uso de biomarcadores permitirá alcançar sensibilidade e especificidade suficientes para a identificação de mulheres suscetíveis.

\section{Referências}

1. Laurenti R, Jorge MHPM, Gotlieb SLD. A mortalidade materna nas capitais brasileiras: algumas características e estimativa de um fator de ajuste. Rev Bras Epidemiol. 2004;7(4):449-60.

2. Saftlas AF, Olson DR, Franks AL, Atrash HK, Pokras R. Epidemiology of preeclampsia and eclampsia in the United States, 1979-1986. Am J Obstet Gynecol. 1990; 163(2):460-5.

3. Geographic variation in the incidence of hypertension in pregnancy. World Health Organization International Collaborative Study of Hypertensive Disorders of Pregnancy. Am J Obstet Gynecol. 1988; 158(1):80-3.

4. Gaio DS, Schmidt MI, Duncan BB, Nucci LB, Matos MC, Branchtein L. Hypertensive disorders in pregnancy: frequency and associated factors in a cohort of Brazilian women. Hypertens Pregnancy. $2001 ; 20(3): 269-81$.

5. Brosens IA, Robertson WB, Dixon HG. The role of the spiral arteries in the pathogenesis of preeclampsia. Obstet Gynecol Annu. 1972;1:177-91.

6. Hanretty KP, Rubin PC. The use of Doppler ultrasound in the study of fetal cardiovascular physiology. J Neurosci Methods. 1990;34(13): $159-67$

7. Roberts JM, Taylor RN, Goldfien A. Clinical and biochemical evidence of endothelial cell dysfunction in the pregnancy syndrome preeclampsia. Am J Hypertens. 1991;4(8):700-8.

8. Roberts JM, Taylor RN, Musci TJ, Rodgers GM, Hubel CA, McLaughlin MK. Pre-eclampsia: a disorders of the endothelial cells? Gynakologe. $1992 ; 25(1): 2-6$

9. Damsky CH, Fitzgerald ML, Fisher SJ. Distribution patterns of extracellular matrix components and adhesion receptors are intricately modulated during first trimester cytotrophoblast differentiation along the invasive pathway, in vivo. J Clin Invest. 1992;89(1):210-22.

10. Damsky CH, Librach C, Lim KH, Fitzgerald ML, McMaster MT, Janatpour M, et al. Integrin switching regulates normal trophoblast invasion. Development. 1994;120(12):3657-66.

11. Fisher SJ. Damsky CH. Human cytotrophoblast invasion. Semin Cell Biol. 1993;4(3):183-8.

12. Pridjian G, Puschett JB. Preeclampsia. Part 1: clinical and pathophysiologic considerations. Obstet Gynecol Surv. 2002;57(9):598-618.

13. Harrington K, Carpenter RG, Goldfrad C, Campbell S. Transvaginal Doppler ultrasound of the uteroplacental circulation in the early prediction of pre-eclampsia and intrauterine growth retardation. Br J Obstet Gynaecol. 1997;104(6):674-81.

14. Papageorghiou AT, Yu CK, Bindra R, Pandis G, Nicolaides KH; Fetal Medicine Foundation Second Trimester Screening Group. Multicenter screening for pre-eclampsia and fetal growth restriction by transvaginal uterine artery Doppler at 23 weeks of gestation. Ultrasound Obstet Gynecol. 2001;18(5):441-9.

15. Phupong V, Dejthevaporn T. Predicting risks of preeclampsia and small for gestational age infant by uterine artery Doppler. Hypertens Pregnancy. 2008;27(4):387-95. 
16. Roncaglia N, Crippa I, Locatelli A, Cameroni I, Orsenigo F, Vergani P, et al. Prediction of superimposed preeclampsia using uterine artery Doppler velocimetry in women with chronic hypertension. Prenat Diagn. 2008;28(8):710-4.

17. Report of the National High Blood Pressure Education Program Working Group on High Blood Pressure in Pregnancy. Am J Obstet Gynecol. 2000; 183(1):S1-S22.

18. Mütze S, Rudnik-Schöneborn S, Zerres K, Rath W. Genes and the preeclampsia syndrome. J Perinat Med. 2008;36(1):38-58.

19. Moncada S, Higgs A. The L-arginine-nitric oxide pathway. N Engl J Med. 1993;329(27):2002-12.

20. Cockell AP, Poston L. Flow-mediated vasodilatation is enhanced in normal pregnancy but reduced in preeclampsia. Hypertension. $1997 ; 30(2$ Pt 1):247-51.

21. Lowe DT. Nitric oxide dysfunction in the pathophysiology of preeclampsia. Nitric Oxide. 2000;4(4):441-58.

22. Anumba DO, Robson SC, Boys RJ, Ford GA. Nitric oxide activity in the peripheral vasculature during normotensive and preeclamptic pregnancy. Am J Physiol. 1999;277(2 Pt 2):H848-54.

23. Ranjan V, Xiao Z, Diamond SL. Constitutive NOS expression in cultured endothelial cells is elevated by fluid shear stress. Am J Physiol. 1995;269(2 Pt 2):H550-5.

24. Hayashi T, Yamada K, Esaki T, Mutoh E, Iguchi A. Effect of estrogen on isoforms of nitric oxide synthase: possible mechanism of anti-atherosclerotic effect of estrogen. Gerontology. 1997;43 Suppl 1:24-34.

25. Jablonka-Shariff A, Olson LM. Hormonal regulation of nitric oxide synthases and their cell-specific expression during follicular development in the rat ovary. Endocrinology. 1997;138(1):460-8.

26. Baylis $C$, Beinder E, Sütö T, August P. Recent insights into the roles of nitric oxide and renin-angiotensin in the pathophysiology of preeclamptic pregnancy. Semin Nephrol. 1998;18(2):208-30.

27. Kleinbongard P, Dejam A, Laver T, Rassaf T, Schindler A, Picker O, et al. Plasma nitrite reflects constitutive nitric oxide synthase activity in mammals. Free Radic Biol Med. 2003;35(7):790-6.

28. Sandrim VC, Palei AC, Cavalli RC, Araújo FM, Ramos ES, Duarte G, et al. eNOS haplotypes associated with gestational hypertension or preeclampsia. Pharmacogenomics. 2008;9(10):1467-73.

29. Founds SA, Dorman JS, Conley YP. Microarray technology applied to the complex disorder of preeclampsia. J Obstet Gynecol Neonatal Nurs. 2008;37(2): 146-57.

30. Johnson CJ, Zhukovsky N, Cass AE, Nagy JM. Proteomics, nanotechnology and molecular diagnostics. Proteomics. 2008;8(4):715-30.

31. Gerszten RE, Wang TJ. The search for new cardiovascular biomarkers. Nature. 2008;451 (7181):949-52.

32. Hewitt SM, Dear J, Star RA. Discovery of protein biomarkers for renal diseases. J Am Soc Nephrol. 2004; 15(7):1677-89.

33. O'Riordan E, Goligorsky MS. Emerging studies of the urinary proteome: the end of the beginning? Curr Opin Nephrol Hypertens. 2005; 14(6):579-85. 